\title{
Diffusion in macromolecular crowded media. Monte Carlo simulation of obstructed diffusion vs. FRAP experiments
}

Eudald Vilaseca ${ }^{1}$, Isabel Pastor ${ }^{1}$, Adriana Isvoran $^{2}$, Sergio Madurga ${ }^{1}$, Josep-Lluís Garcés $^{3}$, and Francesc Mas ${ }^{1, *}$

${ }^{I}$ Physical Chemistry Department and Research Institute of Theoretical and Computational Chemistry (IQTCUB) of the University of Barcelona (UB), C/ Martí i Franquès, 1, 08028Barcelona, Catalonia, Spain

${ }^{2}$ Department of Chemistry, University of the West Timisoara, Timisoara, Romania ${ }^{3}$ Chemistry Department, Lleida University (UdL), Lleida, Catalonia, Spain

${ }^{*}$ Corresponding author e-mail: fmas@ub.edu 


\section{ABSTRACT}

The diffusion of tracer particles in 3D macromolecular crowded media has been studied using two methodologies, simulation and experimental, with the aim of comparing their results. Firstly, the diffusion of a tracer in an obstructed 3D lattice with mobile and big size obstacles has been analyzed through a Monte Carlo (MC) simulation procedure. Secondly, Fluorescence Recovery after Photobleaching (FRAP) experiments have been carried out to study the diffusion of a model protein (alpha-chymotrypsin) in in vitro crowded solution where two type of Dextran molecules are used as crowder agents. To facilitate the comparison the relative size between the tracer and the crowder is the same in both studies. The results indicate a qualitative agreement between the diffusional behaviors observed in the two studies. The dependence of the anomalous diffusion exponent and the limiting diffusion coefficient with the obstacle size and excluded volume shows, in both cases, a similar tendency. The introduction of a reduced mobility parameter in the simulation model accounting for the short range tracer-obstacle interactions allows to obtain a quantitative agreement between the limiting diffusion coefficient values yielded by both procedures. The simulation-experiment quantitative agreement for the anomalous diffusion exponent requires further improvements. As far as we know, this is the first reported work where both techniques are used in parallel to study the diffusion in macromolecular crowded media.

Key words: anomalous diffusion; macromolecular crowding; Monte Carlo simulation,; obstructed diffusion; FRAP 


\section{INTRODUCTION}

Diffusion is a basic transport mechanism that is present in a wide range of complex systems including living cells and it has strong connections with a lot of phenomena of crucial importance for sustaining life. Studies of diffusion-controlled reaction of biological macromolecules are usually performed in dilute solutions (in vitro). However, the high concentration of macromolecules in intracellular environments (in vivo) results in non-specific interactions (macromolecular crowding), which have a great influence on the kinetics and thermodynamics of possible reactions that occur in these systems [1-8]. In fact, macromolecular crowding has been shown to alter molecular diffusion both quantitatively and qualitatively [1, 5, 7, 9-10]. Quantitatively, macromolecular crowding reduces the diffusion coefficient as compared to aqueous solutions and qualitatively, diffusional motion could be changed toward anomalous diffusion, this mean time dependent diffusion.

A great deal of information about motion of molecules in living cells has been obtained from intracellular measurements using different experimental techniques [7, 11-30] and from simulations [10, 31-49]. Experimental data are usually obtained by fluorescence recovery after photobleaching (FRAP) [12-14, 16, 18, 20, 28, 30], fluorescence correlation spectroscopy (FCS) [11-23, 26-27, 29] and single particle tracking (SPT) $[15,19,24-25]$ techniques. Nowadays, from these studies there are still unclear explanations regarding transport processes in living systems. However, it is well known that the anomalous diffusion emerges on cytoplasmatic macromolecules and it depends on the size and conformation of the traced particle and on the total protein concentration of the solution [22]. There are even experimental studies which show anomalous protein diffusion in vitro, with the anomalous diffusion exponent decreasing continuously with increasing obstacle concentration and molecular weight $[26,30]$. 
Simulation methods could be useful to help understanding the molecular dynamics in such complex medium. In the literature there are Monte Carlo (MC) simulation studies of diffusion in 2D media [31-32, 34-35, 41, 45, 47, 49] showing that the lateral diffusion is anomalous for short times and normal for long times. This fact implies that the diffusion coefficients depend on time and this dependency is described by scale laws, whose exponents depend, in turn, on the size and mobility of the crowding molecules [31-32, 34, 41, 43, 46-47]. Such simulations have also been performed in $3 \mathrm{D}[10,33,37-38,42-44,48]$ leading to results that are in satisfactory agreement with experimental data. However, biological fluids are more complex than systems usually considered in simulation due to the nonspecific interactions (e.g. obstruction, trapping or hydrodynamic interactions) and it is necessary to obtain welldefined model systems for the further theoretical, respective experimental investigations.

The aim of the present study is to develop a simple and well-defined model system to carry out Monte Carlo simulations of obstructed diffusion, whose results can be compared with those obtained by an experimental technique. With this objective, we have carried out a series of Monte Carlo simulations and FRAP experiments. On the one hand, we have quantified the effect of the volume excluded by mobile obstacles on the diffusion of a tracer particle in a 3D lattice using MC method. And on the other hand, we have quantified the effect of the volume excluded by Dextran molecules used as crowder agents (two different molecular weights were considered) on the diffusion of a model protein (alpha-chymotrypsin) using FRAP. The size ratio between the model protein and the crowding agents in FRAP experiments is similar to the size ratio between the tracer and the obstacles considered in the Monte Carlo simulations. Finally, the results obtained by both methods are directly compared. 
The paper is organized as follows: Methods, Results and Discussion and Conclusions. "Methods" section contains three subsections: a theoretical background explaining the mathematical concepts of anomalous diffusion, a subsection presenting the simulation algorithm and another subsection presenting the FRAP experiment, explaining how we obtain the values of investigated parameters for the quantitative analysis. In the "Results and discussion" section the results obtained by both methodologies Monte Carlo simulation and FRAP experiments are explained, analyzed and compared. The main outcomes of the study are summarized in the "Conclusions" section.

\section{METHODS}

\section{Theoretical background}

A diffusion process taken by a solute in dilute solutions can be described with the wellknown Einstein-Smoluchowski equation for Brownian motion:

$$
\left\langle r^{2}(t)\right\rangle=(2 d) D t
$$

where $d$ is the topological dimension of the medium where the process is embedded and $D$ is its diffusion coefficient [50-52]. In crowded media, typically in vivo and in a great number of in vitro experiments, the existence of different macromolecular species, proteins, nucleic acids, organelles, etc., hinders the diffusion process. In these cases, Eq. 1 must be generalized to a more complex process, known as anomalous diffusion [11, 26, 51-52] which can be described by:

$$
\left\langle r^{2}(t)\right\rangle=(2 d) \Gamma t^{\alpha}
$$


where $\alpha$ is defined as the anomalous exponent $(0<\alpha<1$ is the case of subdiffusion and $\alpha>1$ holds for the case of superdiffusion) and $\Gamma$ is a generalized transport coefficient, also known as anomalous diffusion coefficient, of units $\left[\right.$ length $^{2} /$ time $\left.^{\alpha}\right]$ whose value depends on the degree of crowding in the medium. This definition allows us to introduce a generalized time-dependent diffusion coefficient function, $D_{\phi}(t)$ as:

$$
D_{\phi}(t) \equiv \frac{1}{(2 d)} \frac{\left\langle r^{2}(t)\right\rangle}{t}=\Gamma t^{\alpha-1}
$$

where $\phi$ represents the excluded volume given by the different macromolecular species present in the solution and determines its crowding degree. Then, Eq. 2 can be written as:

$$
\left\langle r^{2}(t)\right\rangle=(2 d) D_{\phi}(t) t
$$

In order to work with dimensionless magnitudes it is usual to introduce some characteristic length unit, $\lambda$, which is related to the mean free path of the solute and it can be associated to the unit length of the simulation lattice, and some characteristic time unit, $\mu$, which is the jump time and it can be associated to the unit time. Therefore, Eq. 4 becomes:

$$
\left\langle\tilde{r}^{2}(t)\right\rangle=\tilde{D}_{\phi} \tilde{t}
$$

where, $\tilde{r} \equiv r / \lambda$ and $\tilde{t} \equiv t / \mu$ are the dimensionless length and the dimensionless time, respectively, $\tilde{D}_{\phi} \equiv D_{\phi} / D_{0}$, is the dimensionless time-dependent diffusion coefficient function, and $D_{0}$ is the diffusion coefficient of the solute in solution without crowding. This $D_{0}$ value is related to the units of length and time by the Einstein-Smoluchowski Eq. 1 as $D_{0}=(1 / 2 d)\left(\lambda^{2} / \mu\right)$. 
From now, in order to simplify the notation, we drop the tilde in $\tilde{r}, \tilde{t}$ and $\tilde{D}_{\phi}$. Then, Eq. 5 is now written as

$$
\left\langle r^{2}(t)\right\rangle=D_{\phi} t
$$

which is the dimensionless form of Eq. 4. It must be taken into account that the $2 d$ factor only appears in the dimensioned form of the generalized Einstein-Smoluchowski diffusion equation.

Experimental and theoretical data $[10,18,20,22-23,26,34-37,48]$ reveal that, in crowded media, there is a succession of diffusion behaviors that can be identified with the three distinct regions observed in the $\log \left(\left\langle r^{2}\right\rangle / t\right)$ versus $\log (t)$ plots:

i) For really short times $\log \left(<r^{2}>/ t\right)$ is almost time independent reflecting that the diffusion process is not yet affected by macromolecular crowding. We define this initial value as

$$
D_{0}(\phi)=\lim _{t \rightarrow 0} D_{\phi}(t)
$$

and the dimensionless value of $D_{0}(\phi=0)=1$ is in accordance with the $D_{0}$ value for the case without crowding.

ii) Anomalous diffusion corresponds to an intermediate region where $\log \left(<r^{2}>/ t\right)$ linearly decreases with a slope $\alpha-1$.

iii) For long times, $\log \left(<r^{2}>/ t\right)$ tends to be constant again, reflecting a normal diffusion in a homogeneous dense medium with a diffusion coefficient $\left(D^{*}\right)$ lower than that corresponding to a dilute solution 


$$
D^{*}(\phi)=\lim _{t \rightarrow \infty} D_{\phi}(t)
$$

and the dimensionless value of $D^{*}(\phi=0)=1$ is in accordance with the $D_{0}$ value for the case without crowding.

The shifting from the anomalous diffusion regime to the normal one is characterized by the crossover time, $\tau$. These three parameters, $\alpha, \tau$ and $D^{*}$, are considered in our quantitative analysis of diffusion.

\section{Simulation algorithm}

Diffusion in 3D obstructed media has been modeled as a random walk process in which the randomly distributed diffusing particles, called tracers, move in an $80 \times 80 \times 80$ cubic lattice with cyclic boundary conditions and containing randomly distributed obstacles such as their density is under the percolation threshold. We consider only excluded volume interactions (hard-sphere repulsions), so any site in the lattice may not be occupied by two particles at the same time. Our approach is based on the experimental results obtained by Kao and coworkers [33] and Wachsmuth and coworkers [20], which have shown that probe collisions with intracellular components were the principal diffusive barriers that slowed the translational diffusion of small solutes.

Each tracer occupies a single site in the lattice. In contrast, to account for the usual greater size of the crowding molecules and to analyze the effect of this size in the diffusion, the obstacles were considered to occupy a greater number of sites. Two different sizes have been considered for obstacles (see Fig 1): obstacles occupying 27 sites (a $3 \times 3 \times 3$ site cube) and obstacles with 179 sites (a $7 \times 7 \times 7$ site cube with the edge and vertex sites removed to obtain a quasi-spherical shape). As every obstacle occupies 
several sites within the lattice, in our calculations we distinguish the density of sites occupied by obstacles from the concentration of obstacle particles. We will refer to the density of sites occupied by obstacles as the excluded volume due to the obstacle presence $(\phi)$. Four values for the obstacle excluded volume have been considered: $0.031,0.062,0.124$ and 0.187 . We have chosen these densities in agreement with experimental data concerning to the range concentration of macromolecular crowding agents in cytoplasm, 0.05-0.4 [54-55]. We must underline that because of the repulsive interactions between the tracers, the total excluded volume of the system is the sum of $\phi$ plus the volume occupied by the tracers. However, as the tracer excluded volume is the same in all the performed simulations (0.01) and smaller than the obstacle one, all the study will we referred to the obstacle excluded volume, $\phi$.

In all the simulations the obstacles are allowed to move, in order to describe the mobility of the crowded agents in our FRAP experiments. This mobility is controlled by a probability factor $M$ that determines whether an obstacle is tried to move after being randomly selected, being its value related to the obstacle size. The values of 0.75 for 27 site obstacles $(3 \times 3 \times 3)$ and 0.1 for 179 site obstacles (trunqued $7 \times 7 \times 7)$ were employed.

At every time step a particle was selected at random to be moved (a tracer or an obstacle). For a tracer, the destination site is randomly chosen among the 6 nearest neighbors of the origin site. If the proposed site is empty the considered particle moves to it, otherwise it remains in its initial position and another particle is randomly chosen to move. For a big size obstacle, the central site is proposed to move randomly one position in one of the six spatial directions. The obstacle displacement is done if the new sites to be occupied are empty. For each Monte Carlo time step this sequence is repeated $\mathrm{N}_{\text {tot }}$ times $\left(\mathrm{N}_{\text {tot }}\right.$ is the total number of mobile particles within the lattice) in order to assure that statistically each molecule moves once during a time step. Every 
simulation run lasted 10000 time steps and it was repeated from 400 to 800 times with a different initial particle disposition. For each case, the mean squared displacements are averaged along these repetitions. This algorithm was implemented in a Fortran program.

The time dependence of the diffusion coefficient is analyzed, according to Eq. 6, with the $\log \left(<r^{2}>/ t\right)$ versus $\log (t)$ curves obtained from the computer simulations. From each curve the three characteristic parameters of the diffusion can be extracted: the anomalous diffusion exponent $(\alpha)$, the crossover time $(\tau)$ and the long time diffusion coefficient $\left(D^{*}\right)$. In Fig 2 it is shown how these parameters are obtained.

We notice in Fig 2 that for an unobstructed lattice $(\phi=0$, homogenous media) the plot is a horizontal line, indicating that diffusion coefficient is constant. The $\log \left(<r^{2}>/ t\right)$ value is not exactly zero because there is a small auto-crowding effect due to the hard sphere tracer repulsions. In contrast, the curve corresponding to the obstructed lattice $(\phi=0.187)$ present two characteristic regions. There is a region with a linear decreasing of $\log \left(<r^{2}>/ t\right)$, which corresponds to an anomalous diffusion behavior, followed by a region with a smaller constant diffusion coefficient $\left(D^{*}\right)$ characteristic of normal diffusion. It should be noticed that, as a consequence of the spatial discretization of the model $[11,31,36,48]$, the initial region of normal diffusion (when the diffusing particles are not still affected by the crowding obstacles) is not observed in the present simulations. The initial value of $\log \left(<r^{2}>/ t\right)$ is conditioned by the degree of crowding of the system and its linear decreasing is attained after a few time steps. These initial points have been removed from the linear fitting to obtain the anomalous diffusion exponent [35].

According to Eq. 6, the value of the anomalous diffusion exponent is calculated from the slope of the linear time decreasing region of the $\log \left(<r^{2}>/ t\right)$ versus $\log (t)$ plot. The long time diffusion coefficient is the long time asymptotic limit of the plot. Finally, 
as it is illustrated in Fig 2, the crossover time is given by the intersection of the linear fitting of the anomalous diffusion region and the horizontal line corresponding to the limiting diffusion coefficient of the normal diffusion region [35].

The $\left\langle r^{2}>\right.$ versus $t$ plots have also been analyzed. According to Eq. 2, for the normal diffusion regions this plot is a straight line with slop proportional to its corresponding normal diffusion coefficient. As, in the simulations, the crossover time from the anomalous to the final normal diffusion regions occurs very early (in the 28250 time step interval), most of the plot corresponds to the straight line of the final normal diffusion region (Fig 3). The initial normal and anomalous diffusion regions occupy a very short interval. This is the reason why a linear fitting in these plots usually yields the value of the limiting diffusion coefficient $\left(D^{*}\right)$. We have used these plots to confirm the value of the limiting diffusion coefficient $\left(D^{*}\right)$ obtained from Fig 2 . As it will be discussed below, Fig 3 also shows that for a fixed obstacle excluded volume the slop of the $<r^{2}>$ versus $t$ plot changes with the size of the obstacles.

\section{FRAP experiments}

To have comparable experimental results we have to choose an experimental technique which is able to study the properties of a tracer particle (e.g. protein) in a solution with a high concentration of other macromolecules. Among these techniques, those using fluorescent molecules, like Fluorescence Correlation Spectroscopy (FCS) [11-23, 26-27, 29, 53, 56-60] and Fluorescence Recovery after Photobleaching (FPR or FRAP) $[12-14,16,18,20,28,30,41,61-69]$ stand out. In this study we have used FRAP because of its special usefulness for studying molecular dynamics, mainly diffusion processes. 
FRAP curves were acquired using a Leica TCS SP2 UV scanning confocal microscope equipped with a FRAP software package. The experiments were carried out with a $\times 63,1.25 \mathrm{NA}$ water-immersion objective, using a $488 \mathrm{~nm} \mathrm{Ar}^{+}$laser line at $25^{\circ} \mathrm{C}$ and a $8 \%$ of relative intensity. Fluorescence emission was collected using the 500-530 nm band pass filter. Photobleaching illumination was performed using a 476, 488, 496 and $514 \mathrm{~nm} \mathrm{Ar}^{+}$laser line at $25^{\circ} \mathrm{C}$ and a $100 \%$ of relative intensity to bleach a circular region-of interest (ROI) with a diameter of $4.1 \mu \mathrm{m}$. All images were acquired at $512 \times$ 512 pixel resolution, and using a $22.5 \mu \mathrm{m}$ pinhole. The total ROI intensity was collected as a function of time, at increments of $0.28 \mathrm{~ms}$ during $70 \mathrm{~s}$, and measurements were repeated 6 times for each sample. Our samples were composed of a low concentration $\left(8.5510^{-6} \mathrm{M}\right)$ of the FITC-alpha-chymotrypsin complex (alpha-chymotrypsin was labeled with FITC using a manufacturer's protocol) diffusing in an aqueous buffer (phosphate buffered $\mathrm{pH}=7.4$ ) in which crowding agents were dissolved. The concentration of Dextran in the samples is up to $300 \mathrm{mg} / \mathrm{mL}$, in order to have the same excluded volume (from 0 to 0.2 ) as in our Monte Carlo simulations. The selected crowding agents were two Dextran with different sizes: a Dextran with $M_{w}=48600 \mathrm{Da}$ (D1) and a Dextran with $M_{w}=409800$ Da (D2). Samples of $30 \mu 1$ were placed in a spherical cavity microscope slide and were equilibrated for $15 \mathrm{~min}$ on a temperatureregulated microscope stage at $25^{\circ} \mathrm{C}$. In these experimental conditions the contribution to the recovery from diffusion along axial direction is negligible [70], thus the diffusional medium was considered as $2 \mathrm{D}$. This assumption is fulfilled when the bleached area forms a near cylindrical shape through the sample, as it occurs in a circular bleach spot of a reasonable diameter [71]. This assumption simplifies the curve analysis.

FRAP data were fitted with a versatile expression for subdiffusion in bulk solution [30]: 
$F(t)=\left(F_{\infty}-F_{0}\right)\left\{\exp \left(-2\left(\tau_{D} / t\right)^{\alpha}\right)\left[I_{0}\left(-2\left(\tau_{D} / t\right)^{\alpha}\right)+I_{1}\left(-2\left(\tau_{D} / t\right)^{\alpha}\right)\right]\right\}+F_{0}$

where $F(t)$ is the normalized mean fluorescence intensity in the bleached ROI at time $t$; $F_{\infty}$ is the recovered fluorescence at time $t=\infty ; F_{0}$ is the bleached fluorescence intensity at time $t=0 ; I_{0}$ and $I_{1}$ are the modified Bessel Functions; $\tau_{\mathrm{D}}$ is a characteristic residence time of the solute molecule in a volume of a characteristic length $\omega$ (beam area) and $\alpha$ is the anomalous coefficient.

The parameter $\tau_{\mathrm{D}}$, obtained from FRAP experiments, can be related to the generalized transport coefficient $\Gamma$, introduced in Eq. 2

$$
\Gamma=\frac{\omega^{2}}{(2 d) \tau_{D}^{\alpha}}
$$

This definition allows to obtain the generalized time-dependent diffusion coefficient function, $D_{\phi}(t)$, defined in Eq. 3, as:

$$
D_{\phi}(t) \equiv \Gamma t^{\alpha-1} \equiv D^{e f f}\left(\frac{t}{\tau_{D}}\right)^{\alpha-1}
$$

which can be written in terms of an apparent/effective diffusion coefficient, $D^{\text {eff }}$ defined as [26]:

$$
D^{e f f} \equiv D_{\phi}\left(\tau_{D}\right)=\frac{1}{(2 d)} \frac{\omega^{2}}{\tau_{D}}
$$

MATLAB (The Mathworks, Natck, MA) was used to develop the routine to fit experimental data and extract the time constant, $\tau_{\mathrm{D}}$, and the anomalous coefficient, $\alpha$. The goodness of the fitting was judged in terms of $\chi^{2}$ value and weighted residuals. 


\section{RESULTS AND DISCUSSION}

\section{Monte Carlo simulation results}

A group of simulations was carried out in order to analyze two different aspects of the crowding effect on the diffusion of a tracer particle: the effect of the obstacle excluded volume (different occupied volumes) and, for a fixed value of the excluded volume, the effect of the spatial distribution of the occupied sites (different obstacle sizes). It is important to emphasize that in all simulations, obstacles are allowed to move as it happens in FRAP experiments. As in experimental systems the mobility of crowding molecules is proportional to their size, we have analyzed the obstacle obstructive effect to diffusion by assigning a different mobility to each obstacle size. To obtain a qualitative indication of the different size-mobility effect, a value of $M=0.1$ have been given to the 179 site obstacles $(7 \times 7 \times 7)$ and $M=0.75$ for obstacles having 27 sites (3x3x3). In a previous study [48] we have established that the mobility of obstacle chosen are appropriates. The diffusion characteristic parameters obtained from the simulation curves are shown in Fig 4.

First, Fig 4a shows the dependence of the anomalous coefficient, $\alpha$, with the excluded volume by the two selected obstacle sizes. In both cases a consistent decrease of $\alpha$ for the tracer particle is observed when the concentration of both obstacles is increased. In addition, it can be seen that for each obstacle excluded volume, the anomalous diffusion exponent is greater for the small size obstacles and smaller for the big ones. Second, the limiting diffusion coefficient, $D^{*}$ (Fig. 4b), shows a similar behavior: a decrease of the value of $D^{*}$ is observed when the concentration of both obstacles is increased. Moreover, despite that the $D^{*}$ value differences are small, for a given obstacle excluded volume; the bigger obstacles $(7 \times 7 \times 7)$ have a smaller diffusion coefficients. 


\section{FRAP results}

Figure 5 shows an example of the FRAP curves obtained in our experiments. We could use successfully the analysis model proposed in Eq. 10 to fit these experimental data and to characterize the diffusional behavior of the protein alpha-chymotrypsin in samples with the two considered Dextrans (D1 and D2). The values collected in Table 1 show an increase of the diffusional time $\tau_{\mathrm{D}}$ and a consistent decrease of the anomalous coefficient $\alpha$ for the protein when the excluded volume (i.e. concentration) of both Dextrans in solution is increased. Moreover, we can see a size-dependent emergence of anomalous subdiffusion, which also clearly depends on the fractional volume occupied by the crowding agent. These experiments confirm that the interaction via excluded volume can cause subdiffusion. In addition, in Fig. 6 we can see that $\alpha$ decays with increasing obstacle concentration and this decay becomes steeper with increasing the obstacle size (average molecular weight of Dextran). This behavior is similar than the obtained in Monte Carlo simulations.

From the parameters obtained in FRAP experiments and using Eq. 13, it is possible to calculate an effective diffusion coefficient, $D^{\text {eff }}$, for FITC-alphachymotrypsin in Dextran solutions. The obtained values for the different Dextran concentrations are also shown in Table 1 . As a reference, $D^{\text {eff }}$ for alpha-chymotrypsin in

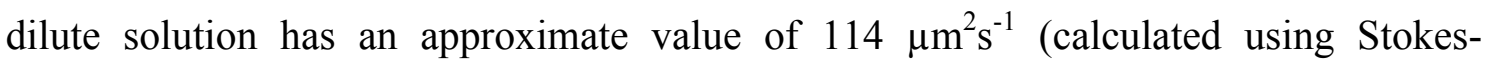
Einstein equation). In these tables we can see that $D^{\text {eff }}$ has a similar behavior as the anomalous diffusion parameter $\alpha$, this means that $D^{\text {eff }}$ decreases with increasing concentration and size $\left(M_{w}\right)$ of obstacles.

As the time scale of confocal FRAP experiments is very short, we could observe the time-dependence of the diffusion coefficient of alpha-chymotrypsin in crowded Dextran solutions (Fig. 7) using Eq. 4, and it was also possible to obtain its limiting 
value corresponding to the long time normal diffusion, $D^{*}=D^{e f f} / D_{0}$. To calculate this limiting diffusion coefficient, $D^{*}$, we had to consider the experimental initial diffusion parameter, $D_{0}$, which is different from the diffusion coefficient of the protein in a dilute solution. At $t \rightarrow 0$ the diffusion coefficient is not exactly the one corresponding to a dilute solution because the properties of water molecules confined in reduced spaces are not the same. It is known, for example, that the effective viscosity of water due to the presence of larges obstacles can increase up to 20 times [72-73]. This fact suggests that, according to Eq. 7, the value of $D_{0}$ that we have to take into account in each sample is its corresponding initial diffusion coefficient value. The obtained values of $D^{*}$ are shown in Table 1 too. It is observed a decrease of $D^{*}$ when the concentration of both Dextrans is increased. In addition, for a given excluded volume, the bigger Dextran has a smaller diffusion coefficient. This behavior is also similar to that obtained in the Monte Carlo simulation.

\section{Comparison}

Table 2 shows the values of $\alpha$ and $D^{*}$ obtained through both methodologies, Monte Carlo simulations and FRAP experiments, in function of the excluded volume and the obstacle size. The results indicate that both techniques describe a similar diffusional behavior of the system. We observe a time dependent diffusion (anomalous subdiffusion) with the two methodologies. We can also see that $\alpha$ and $D^{*}$ decay with increasing obstacle concentration and that this decay becomes steeper with increasing the obstacle size. Both, simulation and experimental, studies indicate that the size and the volume excluded by obstacles play a very important role in the diffusion processes in macromolecular crowded media, concluding that they must be taken into account in future studies on diffusion-controlled processes, such as reactions in crowded media. 
However, it should be noted that there are quantitative differences between the results obtained by Monte Carlo simulations and those obtained by FRAP experiments. Generally, for both parameters reported in the Table 2 the values obtained by FRAP are lower than those obtained through Monte Carlo simulations, indicating that this procedure yields a weaker excluded volume effect. In view of these results, it is not clear that the MC simulation algorithm introduced here can reproduce the anomalous diffusion mechanism associated with the experimental FRAP curves.

Several mechanisms are proposed in the literature to describe the subdiffusion process of macromolecules in crowded media. They can be summarized in three different kinds [41, 52, 74-76]: (i) obstructed diffusion; (ii) Continuous Time Random Walk (CTRW); (iii) Fractional Brownian Motion (FBM). The Monte Carlo simulation model presented here belongs to the obstructed diffusion type. FBM considers a timedependent diffusion coefficient similar to that defined in Eq. 3 to interpret the meansquared displacement (MSD) for a diffusion process in an obstructed medium. On the other hand, CTRW considers a random walk process among energetic or structural traps with a random waiting time, which is consistent with the description of the MSD through Eq. 2 with a generalized transport coefficient. This generalized transport coefficient is, in CTRW, non local in time, a property intimately related to its nonstationary nature, in contrast to the FBM where it is local in time.

Recently Weiss et al. has shown that FCS experiments are consistent with obstructed diffusion and FBM descriptions and differs from CTRW [75-76]. Moreover, Tejedor et al. [77] have proposed new estimates from trajectories of particles in order to discriminate the different mechanisms for describing subdiffusion, and they applied them to Single Particle Tracking (SPT) experiments, showing that FBM is the most consistent mechanism that explains the different observed experimental features. 
A FRAP experiment, as performed here, is not sensitive to the various types of individual particle motions, but rather measures ensemble properties. When obtaining FRAP curves, one sees only an average of the different individual behaviors and therefore an apparent diffusion behavior [68] is obtained. On the other hand, the MC simulation algorithm for obstructed diffusion introduced here makes a similar process because the mean-squared displacement (MSD) is obtained by averaging different particle trajectories at different times. Lubelski and Klafter [68] generalized the FRAP response with a Gaussian laser beam consistent with the CRTW mechanism following a subordination relationship between the solutions of the fractional (subdiffusion) and normal diffusion equations, in a similar manner as Feder et al. [11] did using a FBM description of the FRAP experiment with a Gaussian laser beam. Recently, Pastor et al. [30] have made a similar procedure for a FRAP experiment with a uniform laser beam. Although simulations give some discrepancies for cases where there is a fraction of immobile macromolecules between these two mechanisms, for cases when all the macromolecules are assumed mobile, the two procedures seem to give a similar description of the FRAP experiment with Gaussian laser beam [68]. Saxton [41] made simulations of FRAP experiments using the three different mechanisms, above mentioned, and showed that there are some discrepancies between them, especially if there is a fraction of immobile macromolecules/proteins, being FBM the one most consistent.

Moreover, it should be mentioned that all the MC simulations performed using the obstructed diffusion algorithm, are made in the percolation threshold. $[41,76]$ or with a exclude volume compatible with the $\alpha$ value obtained experimentally [75].

According to these analyses, the obstructed diffusion MC model used here is a reliable mechanism to describe anomalous diffusion in crowded media and to interpret 
the information yielded by experimental FRAP curves obtained with a uniform laser beam in a medium without an immobile fraction of macromolecules/proteins. Thus, the observed quantitative discrepancies between results from both the MC and FRAP descriptions can be corrected by introducing some improvements in the simulation model. In particular, the introduction of the different type of interactions (hydrodynamic, esteric, conformational, etc.) that the diffusing macromolecules undergo in their diffusion process can improve the model.

Although the volume excluded by obstacles in the MC simulations is the same as in the experiments, the fact that the tracer-obstacle interaction is described with a hard sphere potential causes its crowding effect being smaller. The consideration of short range tracer-obstacle interactions and the greater viscosity of the solution near an obstacle can improve the comparison of the MC results with the experiments. To do so we have modified the simulation algorithm by introducing a retention parameter for the tracers those are in contact to an obstacle. According to the simulation model, at each time step a number of tracer particles is proposed to move and each particle is moved if the destination position is empty. Until now the movement trial is done for all the selected particles. Now we introduce a probability factor (RedMob) that is 0.5 for the tracers in contact to an obstacle and 1 for the rest of tracers. When a tracer particle is selected at random, the trial movement is done according to this probability factor. So, the mobility of particles touching an obstacle will be reduced as it happens in a real system. The value of 0.5 was selected as a primary estimation of the relative mobility of tracers touching an obstacle with respect to tracers in the bulk.

The simulations with obstacles having a size of 27 sites $(3 \times 3 \times 3)$ were repeated for the 4 obstacle concentrations using this reduced mobility factor (RedMob). The obtained results are shown in Table 2. There it we can see that, now, the crowding effect 
is more important. The limiting diffusion coefficient $\left(D^{*}\right)$ values are much smaller, approaching to the experimental results and showing a similar variation interval. However, the anomalous diffusion coefficient $(\alpha)$ values are exactly the same as in the previous simulations. The reduced mobility factor, does not modify the slope of the $\log \left(<r^{2}>/ t\right)$ versus $\log (t)$ curves. There is a displacement of these curves to lower values. The limiting diffusion coefficient diminishes but the initial diffusion coefficient, $D_{0}(\phi)$, also diminishes in the same proportion (data not shown). Thus, the anomalous diffusion region starts at a new position and the diffusion coefficient variation until reaching the limiting normal diffusion is the same.

It should be noticed that this result indicates that the crowding effect on the diffusion needs the consideration of tracer-obstacle interactions to be interpreted since the exclusive consideration of an excluded volume effect (in the sense of a hard sphere potential) yields insufficient results. Additional improvements should be introduced to the model to obtain a more exact description of the anomalous diffusion exponent value. These improvements must be done in two different ways. On the one hand, by subdiving the cells of the MC simulation lattice into smaller size cells in order to minimize the effect of the spatial and temporal discretization in the final value of the estimated parameters, and, on the other hand, by developing either new off-lattice MC algorithms or adapting Brownian Dynamics (BD) algorithms [78-80], in order to take into account the different kind of interactions mentioned aboveHowever, it is noteworthy that a simple MC model as the one presented here is able to describe the crowding effect on the diffusion with a good level of approximation to reality.

\section{CONCLUSIONS}


In this work we have shown the potentialities of Monte Carlo simulations in the study of diffusion phenomena in macromolecular crowding situations. The obtained results show the important role of the size and the concentration of crowded agents in the diffusion of a tracer molecule in crowded media, and the behavior reported by our simulations is qualitatively similar to that obtained by experimental FRAP measurements. Despite the quantitative discrepancies, the introduction of a reduced mobility factor, representing the tracer-obstacle short range interactions, notably improves the agreement between simulations and experiments. This results indicates that the pure consideration of the excluded volume is not enough to correctly describe the crowding effects on diffusion.

\section{ACKNOWLEDGMENTS}

We acknowledge the financial support from: CNCSIS 551 Romania, Spanish Ministry of Science and Innovation (Projects UNBA05-33-001 and CTM2009-14612) and "Comissionat d'Universitats i Recerca de la Generalitat de Catalunya" (grants 2009SGR465 and XRQTC). IP thanks the Juan de la Cierva Program of the Spanish Ministry of Science. 


\section{REFERENCES}

1. Minton AP (2001) J Biol Chem 276: 10577-10580

2. Ellis RJ (2001) Trends Biochem Sci 26: 597-604

3. Ellis RJ, Minton AP (2003) Nature 425: 27-28

4. Derham BK, Harding JJ (2006) BBA 1764: 1000-1006

5. Zhou HX, Rivas G, Minton AP (2008) Annu Rev Biophys 37: 375-395

6. Agrawal M, Santra SB, Anand R, Swaminathan R (2008) PRAMANA J Phys 71: $359-368$

7. Dix JA, Verkman AS (2008) Annu Rev Biophys 37: 247-263

8. Zhou HX (2009) J Phys Chem B 113: 7995-8005

9. Ben-Avraham D, Havlin S (2000) Diffusion and reactions in fractals and disordered systems. Cambridge University Press, Cambridge

10. Saxton MJ (2007) Biophys J 92: 1178-1191

11. Feder TJ, Brust-Mascher I, Slattery JP, Baird B, Webb WW (1996) Biophys J 70: $2767-2773$

12. Seksek O, Biwersi J, Verkman AS (1997) J Cell Biol 138: 131-142

13. Periasamy N, Verkman AS (1998) Biophys J 75: 557-567

14. Ario-Dupont M, Foucault G, Vacher M, Devaux F, Cribier S (2000) Biophys J 78: $901-907$

15. Platani M, Goldberg I, Swedlow JR, Lamond AI (2000) J Cell Biol 151: 1561-1574

16. Potma EO, de Boeij WP, Bosgraaf L, Roelofs J, Van Haastert PJM, Wiersma DA (2001) Biophys J 81: 2010- 2019

17. Verkman AS (2002) Science 27: 27-33

18. Cheng Y, Prud'homme RK, Thomas JL (2002) Macromolecules 35: 8111- 8121

19. Platani M, Goldberg I, Lamond A I, Swedlow JR (2002) Nat Cell Biol 4: 502-508 
20. Wachsmuth M, Weidemann T, Muller G, Hoffman-Rohrer MW, Knoch TA, Waldeck W, Langowski J (2003) Biophys J 84: 3353-3363

21. Fatin-Rouge N, Starchev K, Buffle J (2004) Biophys J 86: 2710-2719

22. Dauty E, Verkman AS (2004) J Molecular Recognition 17: 441-447

23. Weiss M, Elsner M, Kartberg F, Nilsson T (2004) Biophys J 87: 3518-3824

24. Murase K, Fujiwara T,UmemuraY, Suzuki K, Iino R, et al. (2004) Biophys J 86: $4075-4093$

25. Kusumi A, Ike H, Nakada C, Murase K, Fujiwara T (2005) Semin Immunol 17: 321

26. Banks DS, Fradin C (2005) Biophys J 89: 2960-2971

27. Masuda A, Ushida K, Okamoto T (2005) Biophys J 88: 3584-3591

28. Sanabria H, Kubota Y, Waxhan MN (2007) Biophys J 92: 313-322

29. Guigas M, Kalla C, Weiss M (2007) Biophys J 93: 316-323

30. Pastor I, Vilaseca E, Madurga S, Garcés JL, Cascante M, Mas F (2010) J Phys Chem B 114: 4028-4034

31. Saxton MJ (1987) Biophys J 52: 989-997

32. Saxton MJ (1990) Biophys J 58: 1303-1306

33. Kao HP, Abney JR, Verkman AS (1993) J Cell Biol 120: 175-184

34. Saxton MJ (1993) Biophys J 64: 1053-1962

35. Saxton MJ (1994) Biophys J 66: 394-401

36. Saxton MJ (1996) Biophys J 70: 1250-1262

37. Netz PA, Dorfmuller T (1995) J Chem Phys 103: 9074-9082

38. Olveczki BP, Verkman AS (1998) Biophys J 74: 2722-2730

39. Gil A, Segura J, Pertusa JAG, Soria B (2000) Biophys J 78: 13-33

40. Tang Y, Schlumpberger T, Kim T, Lueker M, Zucker RS (2000) Biophys J 78: $2735-2751$ 
41. Saxton MJ (2001) Biophys J 81: 2226-2240

42. Lipkow K, Andrews SS, Bray D (2005) J Bacteriology 187: 45-53

43. Dix JA, Hom EF, Verkman AS (2006) J Phys Chem B 110: 1896-1906

44. Echeveria C, Tucci K, Kapral R (2007) J Phys Condens Matter 19: 065146-

45. Isvoran A, Vilaseca E, Garces JL, Unipan L, Mas F (2007) Proceedings of the 6th Conference of Balkan Physics Union, A.I.P. 889: 469-470

46. Isvoran A, Vilaseca E, Unipan L, Garces JL, Mas F (2007) Rom Biophys J 17: 21 32

47. Isvoran A, Vilaseca E, Unipan L, Garces JL, Mas F. (2008) Rev Rom Chem 53: $415-419$

48. Vilaseca E, Isvoran A, Madurga S, Garces JL, Pastor I, Mas F. (2010), submitted

49. Guigas G, Weiss M (2008) Biophys J 94: 90-94

50. Havlin S, Ben-Avraham D (1987) Adv Phys 36: 695-798

51. Bouchaud JP, Georges A (1990) Phys Rep 185: 127-293

52. Metzler R, Klafter J (2000) Phys Rep 339: 1-77

53. Franks F (1993) Protein biotechnology. Isolation, characterization and stabilization. Franks F. Editor. Humana Press, Clifton NJ.

54. Fuklton AB (1982) Cell 30: 345-347

55. Zimmerman SB, Minton AP (1993) Annu Rev Biophys Biomol Struct 22: 27-65

56. Fradin C, Abu-Arish A, Granek R, Elbaum M (2003) Biophys J 84: 2005-2020

57. Schwille P, Korlach J, Webb WW (1999) Cytrometry 36: 176-182

58. Szymański J, Patkowki A, Gapiński J, Wilk A, Holyst R (2006) J Phys Chem B 110: $7367-7373$

59. Reitan NK, Juthajan A, Lindmo T, de Lange Davies C (2008) J Biomol Opts 13: 054040- 
60. Cherdhirakorn T, Best A, Koynov A, Peneva K, Muellen K, Fytas G (2009) J Phys Chem B 113: 3355-3359

61. Periasamy N, Verkman AS (1998) Biophys J 75: 557-567

62. Pyenta PS, Schwille P, Webb WW, Holowka D, Baird B (2003) J Phys Chem A 107: $8310-8318$

63. Ratto TV, Longo ML (2003) Langmuir 19: 1788-1793

64. Dunham SM, Pudavar HE, Prasad PN, Stachowiak MK (2004) J Phys Chem B 108: $10540-10546$

65. Heitzman CE, Tu H, Braun PV (2004) J Phys Chem B 108: 13764-13770

66. Pucadyil TJ, Chattopadhyay AJ (2006) Fluorescence 16: 87-94

67. Pucadyil TJ, Mukherjee S, Chattopadhyay A (2007) J Phys Chem B 111: 1975-1983

68. Lubelski A, Klafter J (2008) Biophys J 94: 4646-4653

69. Braga J, Desterro JMP, Carmo-Fonseca M (2004) Mole Biol Cell 15: 4749-4760

70. Blonk JCG, Don A, Aalst HV, Birmingham JJ (1992) J Microsc 169: 363-374

71. Sprague BL, Pego RL, Stavreva DA, McNally JG (2004) Biophys J 86: 3473-3495

72. Lüsse S, Arnold A (1998) 31: 6891-6897

73. Tan HS, Piletic IR, Fayer MD (2005) J Chem Phys 122: 174501-174509

74. Bouchaud JP, Georges A (1990) Phys Rep 195: 127-293

75. Szymanski J, Weiss M (2009) Phys Rev Lett 103: 038102

76. Malchus N, Weiss M (2010) J Fluoresc 20: 19-26

77. Tejedor V, Bénichou O, Voituriez R, Jungmann R, Simmel F (2010) Biophys J 98: $1364-1372$

78. Northrup SH, Allison SA, MaCammon JA (1983) J Chem Phys 80: 1517-1524

79. Norhrup SH, Erickson HP (1992) Proc Natl Acad Sci (USA) 89: 3338-3342

80. Chen JC, Kim AS (2004) Adv Colloid Interfac 112: 159-173 
Table 1. Experimental parameters associated with the diffusion process of alphachymotrypsin as a function of size and concentration of crowding agents assuming a uniform circular disc profile model

\begin{tabular}{|c|c|c|c|c|c|}
\hline Crowder & $\mathrm{C}(\mathrm{mg} / \mathrm{mL})$ & $\tau_{\mathrm{D}}(\mathrm{s})$ & $\alpha$ & $D^{\mathrm{eff}}\left(\mu \mathrm{m}^{2} / \mathrm{s}\right)$ & $D^{*}$ \\
\hline \multirow[t]{4}{*}{ D1 } & 50 & $7.00 \pm 0.31$ & $0.94 \pm 0.04$ & $7.90 \pm 0.41$ & $0.82 \pm 0.13$ \\
\hline & 100 & $8.23 \pm 0.40$ & $0.88 \pm 0.04$ & $6.71 \pm 0.30$ & $0.66 \pm 0.08$ \\
\hline & 200 & $11.27 \pm 0.35$ & $0.90 \pm 0.03$ & $4.91 \pm 0.12$ & $0.68 \pm 0.04$ \\
\hline & 300 & $15.98 \pm 0.25$ & $0.87 \pm 0.03$ & $3.46 \pm 0.08$ & $0.59 \pm 0.03$ \\
\hline \multirow[t]{3}{*}{ D2 } & 50 & $9.45 \pm 0.37$ & $0.90 \pm 0.04$ & $5.85 \pm 0.22$ & $0.68 \pm 0.07$ \\
\hline & 100 & $10.44 \pm 0.27$ & $0.82 \pm 0.02$ & $5.29 \pm 0.08$ & $0.51 \pm 0.02$ \\
\hline & 200 & $13.45 \pm 0.20$ & $0.82 \pm 0.03$ & $4.11 \pm 0.11$ & $0.48 \pm 0.03$ \\
\hline
\end{tabular}


Table 2. Values of $\alpha$ and $D^{*}$ obtained through both methodologies Monte Carlo simulation and FRAP experiments in function of the excluded volume and the size of obstacles. The numerical errors for all the simulation values are smaller than $1 \times 10^{-4}$

\begin{tabular}{|c|c|c|c|c|c|}
\hline & \multirow[b]{2}{*}{$\phi$} & \multicolumn{2}{|c|}{ MC simulations } & \multicolumn{2}{|c|}{ FRAP experiments } \\
\hline & & $\alpha$ & $D^{*}$ & $\alpha$ & $D^{*}$ \\
\hline \multirow{4}{*}{$\begin{array}{c}3 \times 3 \times 3 \\
M=0.75 \text { or } \\
\text { D1 }\end{array}$} & 0.031 & 0.9977 & 0.9663 & $0.94 \pm 0.04$ & $0.82 \pm 0.13$ \\
\hline & 0.062 & 0.9957 & 0.9438 & $0.88 \pm 0.04$ & $0.66 \pm 0.08$ \\
\hline & 0.124 & 0.9915 & 0.8975 & $0.90 \pm 0.03$ & $0.68 \pm 0.04$ \\
\hline & 0.187 & 0.9871 & 0.8521 & $0.87 \pm 0.03$ & $0.59 \pm 0.03$ \\
\hline \multirow{4}{*}{$\begin{array}{c}7 \times 7 \times 7 \\
M=0.1 \text { or } \\
\text { D2 }\end{array}$} & 0.031 & 0.9967 & 0.9650 & $0.90 \pm 0.04$ & $0.68 \pm 0.07$ \\
\hline & 0.062 & 0.9933 & 0.9423 & $0.82 \pm 0.02$ & $0.51 \pm 0.02$ \\
\hline & 0.124 & 0.9863 & 0.8951 & $0.82 \pm 0.03$ & $0.48 \pm 0.03$ \\
\hline & 0.187 & 0.9786 & 0.8477 & - & - \\
\hline \multirow{4}{*}{$\begin{array}{c}3 \times 3 \times 3 \\
M=0.75 \\
\text { RedMob }= \\
0.5 \text { or D1 }\end{array}$} & 0.031 & 0.9976 & 0.9244 & $0.94 \pm 0.04$ & $0.82 \pm 0.13$ \\
\hline & 0.062 & 0.9955 & 0.8607 & $0.88 \pm 0.04$ & $0.66 \pm 0.08$ \\
\hline & 0.124 & 0.9913 & 0.7550 & $0.90 \pm 0.03$ & $0.68 \pm 0.04$ \\
\hline & 0.187 & 0.9872 & 0.6577 & $0.87 \pm 0.03$ & $0.59 \pm 0.03$ \\
\hline
\end{tabular}




\section{CAPTIONS}

FIGURE 1. The two obstacle sizes considered in the MC simulations: 27 sites $(3 \times 3 \times 3)$ and 179 sites $(7 \times 7 \times 7 R)$.

FIGURE 2. $\log \left(<r^{2}>/ t\right)$ versus $\log (t)$ curve for diffusion in a 3D lattice with mobile obstacles having a size of 27 sites $(3 \times 3 \times 3)$ and an excluded volume of $\phi=0.187$. The curve for a lattice without obstacles is also added. The regions corresponding to the different diffusion behaviors and the manner to determine the characteristic parameters are illustrated.

FIGURE 3. Plot of $\left\langle\mathrm{r}^{2}\right\rangle$ versus time in two systems with a same obstacle excluded volume (0.187) but having different obstacle sizes: 27 sites $(3 \times 3 \times 3)$ and 179 sites $(7 \times 7 \times 7 \mathrm{R})$. The plot for the reduced mobility (RedMob) simulations in a lattice with $3 \times 3 \times 3$ obstacles is also included. The arrow indicates the crossover time position.

FIGURE 4. Dependence of a) the anomalous diffusion exponent, $\alpha$, and b) the long time diffusion coefficient, $D^{*}$, on the obstacle excluded volume for two different size obstacles having different mobility: 27 site obstacles $(\mathrm{M}=0.75)$ and 179 site obstacles $(\mathrm{M}=0.1)$.

FIGURE 5. FRAP curve obtained for the solution of alpha-chymotrypsin in a solution with a $50 \mathrm{mg} / \mathrm{mL}$ of $\mathrm{D} 1(\phi=0.031)$.

FIGURE 6. Anomalous diffusion exponent associated with the diffusion of alphachymotrypsin as a function of obstacle concentration for Dextrans of various average molecular weights $(\bullet)$ for D1 and $(\boldsymbol{\Delta})$ for D2. 
FIGURE 7. Plots obtained using Eq. 13 that show the time dependence of the diffusion coefficient associated with the diffusion of alpha-chymotrypsin for different obstacle concentrations of both used Dextran: (匹) $50 \mathrm{mg} / \mathrm{mL}$, (о) $100 \mathrm{mg} / \mathrm{mL},(\boldsymbol{\Delta}) 200 \mathrm{mg} / \mathrm{mL}$ and $(\nabla) 300 \mathrm{mg} / \mathrm{mL}$. 
FIGURE 1
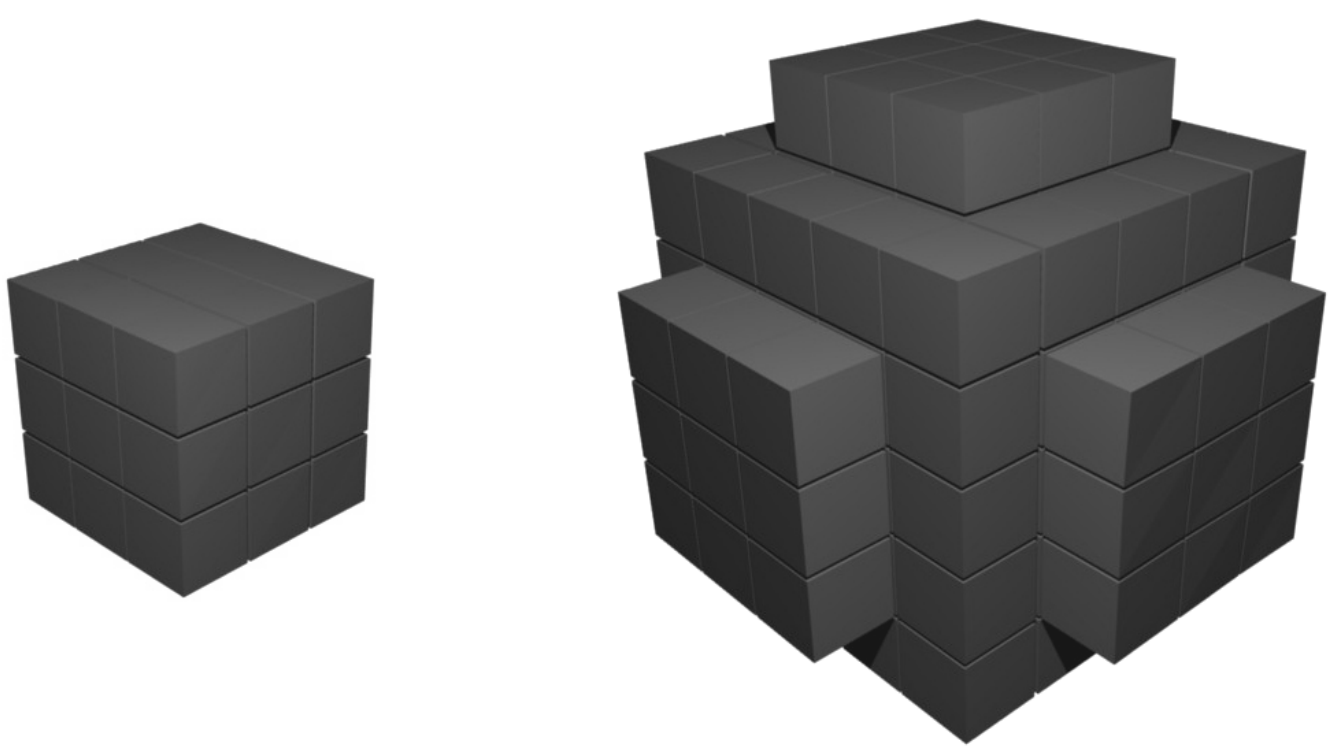
FIGURE 2

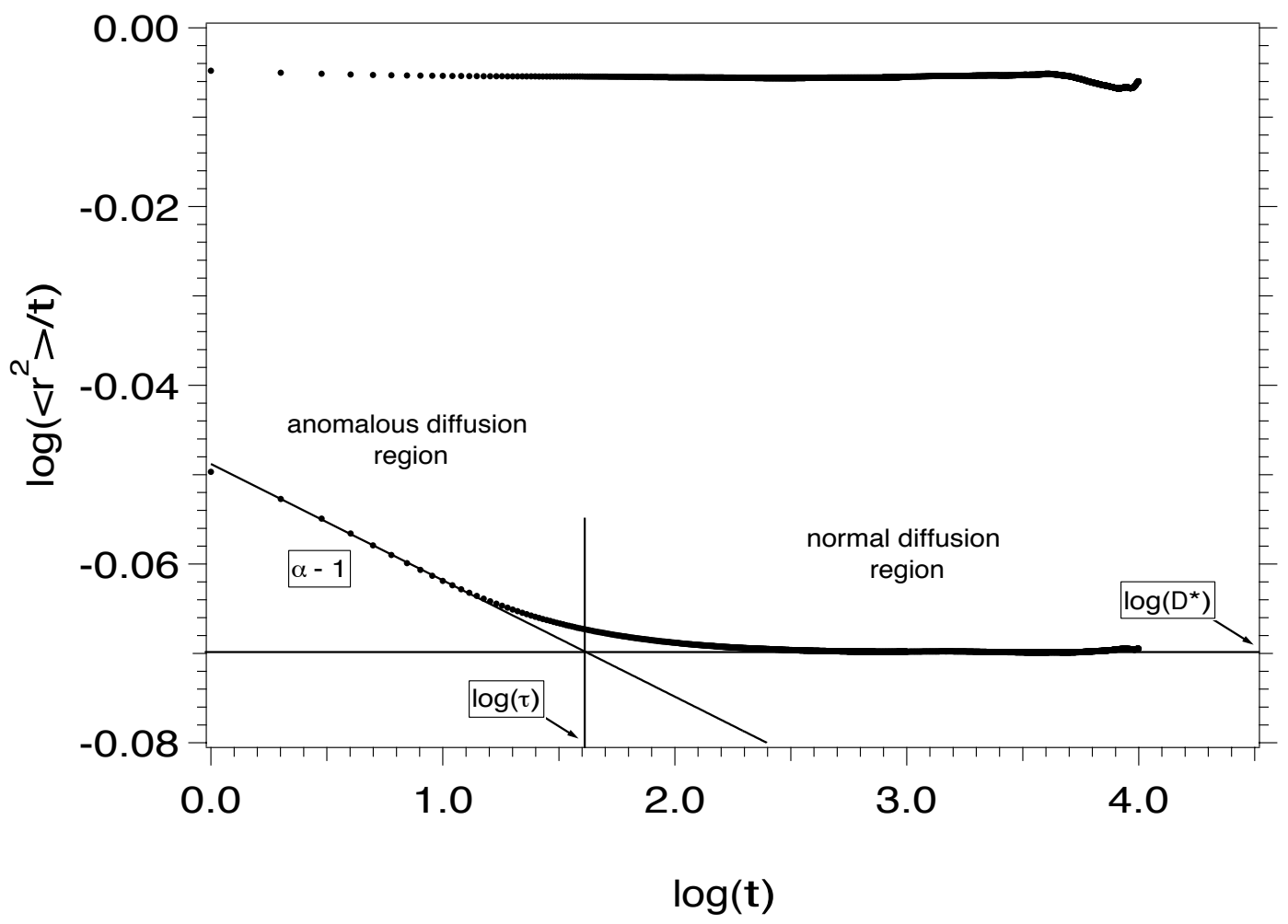


FIGURE 3

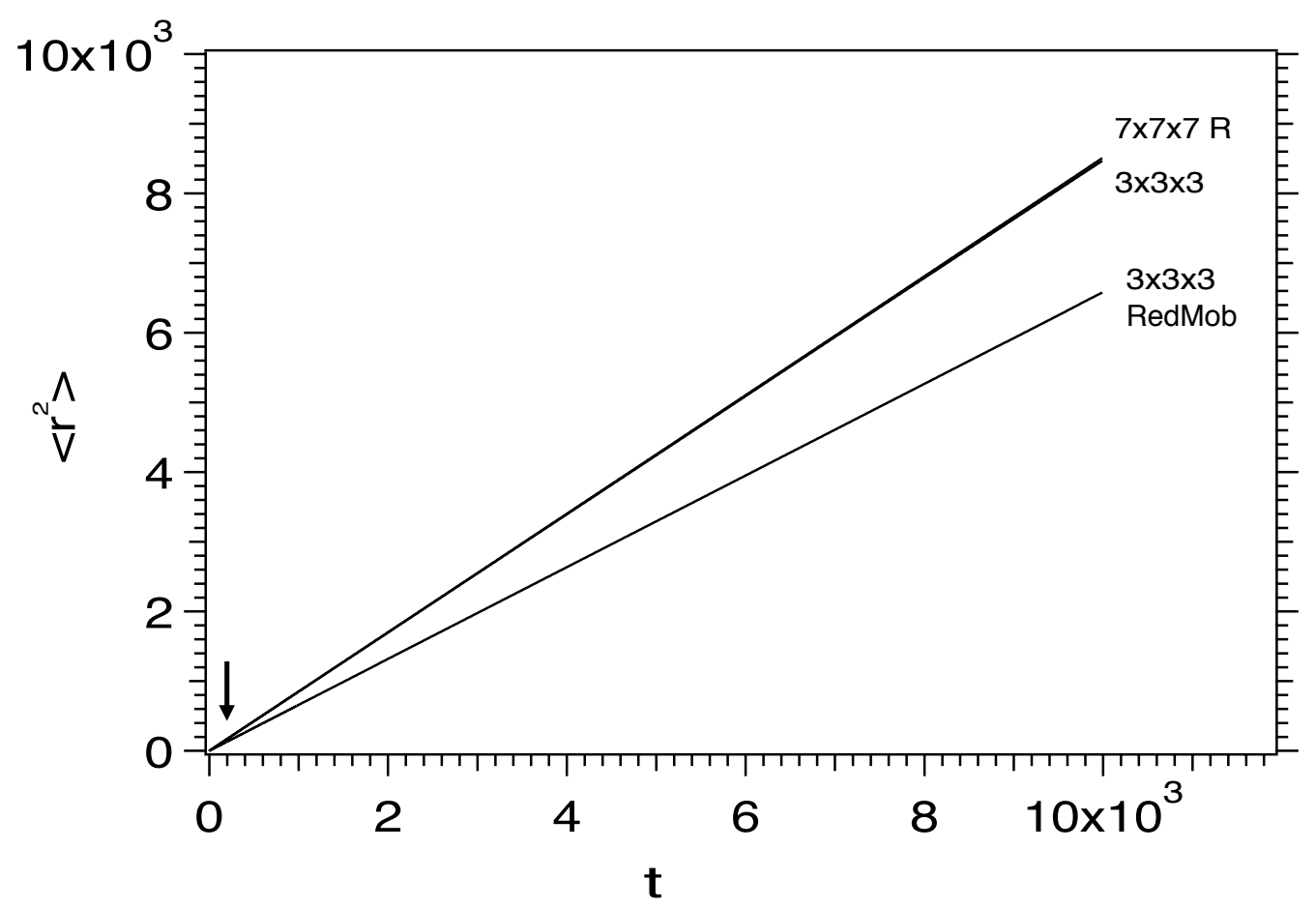


FIGURE 4
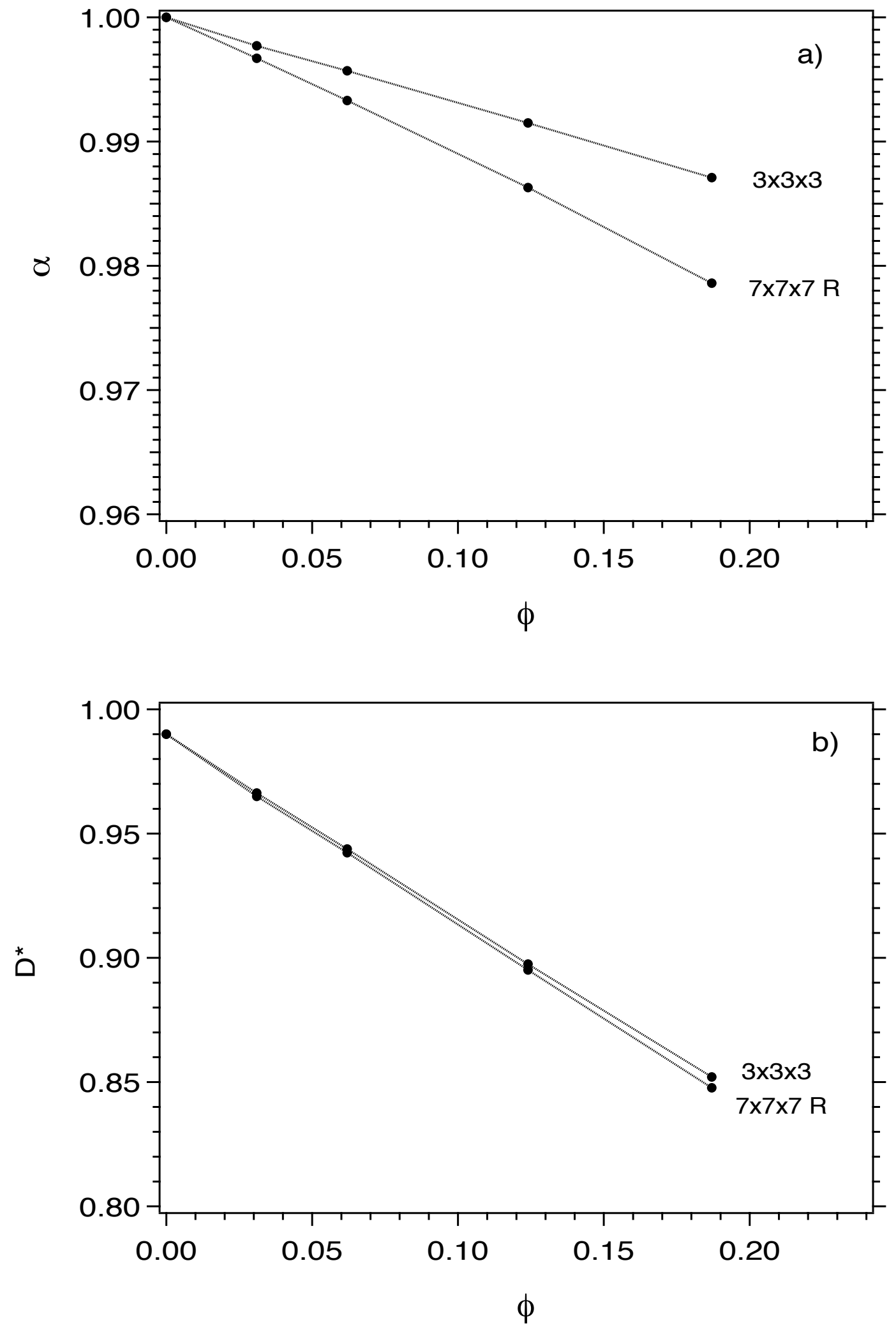
FIGURE 5

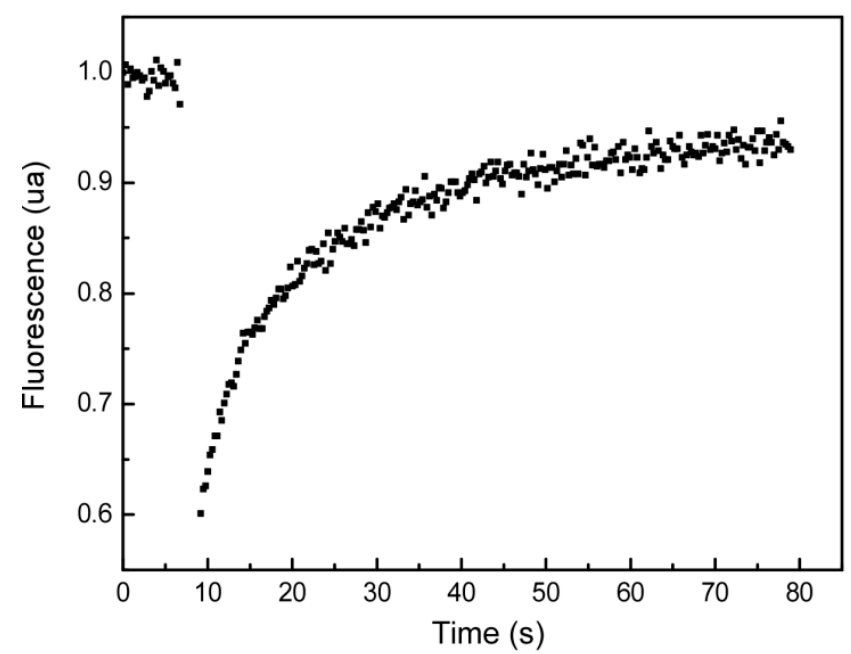


FIGURE 6

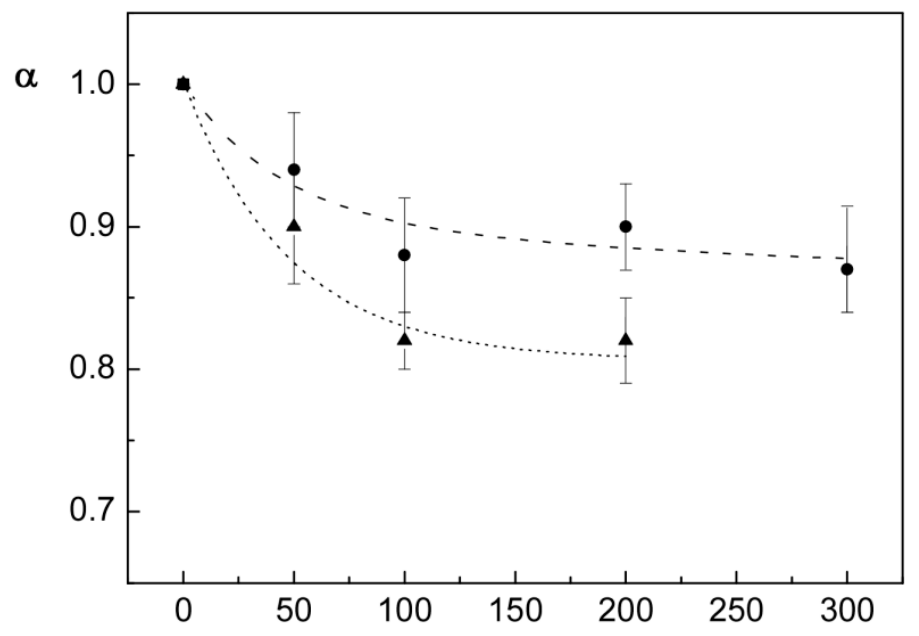

[Dextran] (mg/mL) 


\section{FIGURE 7}
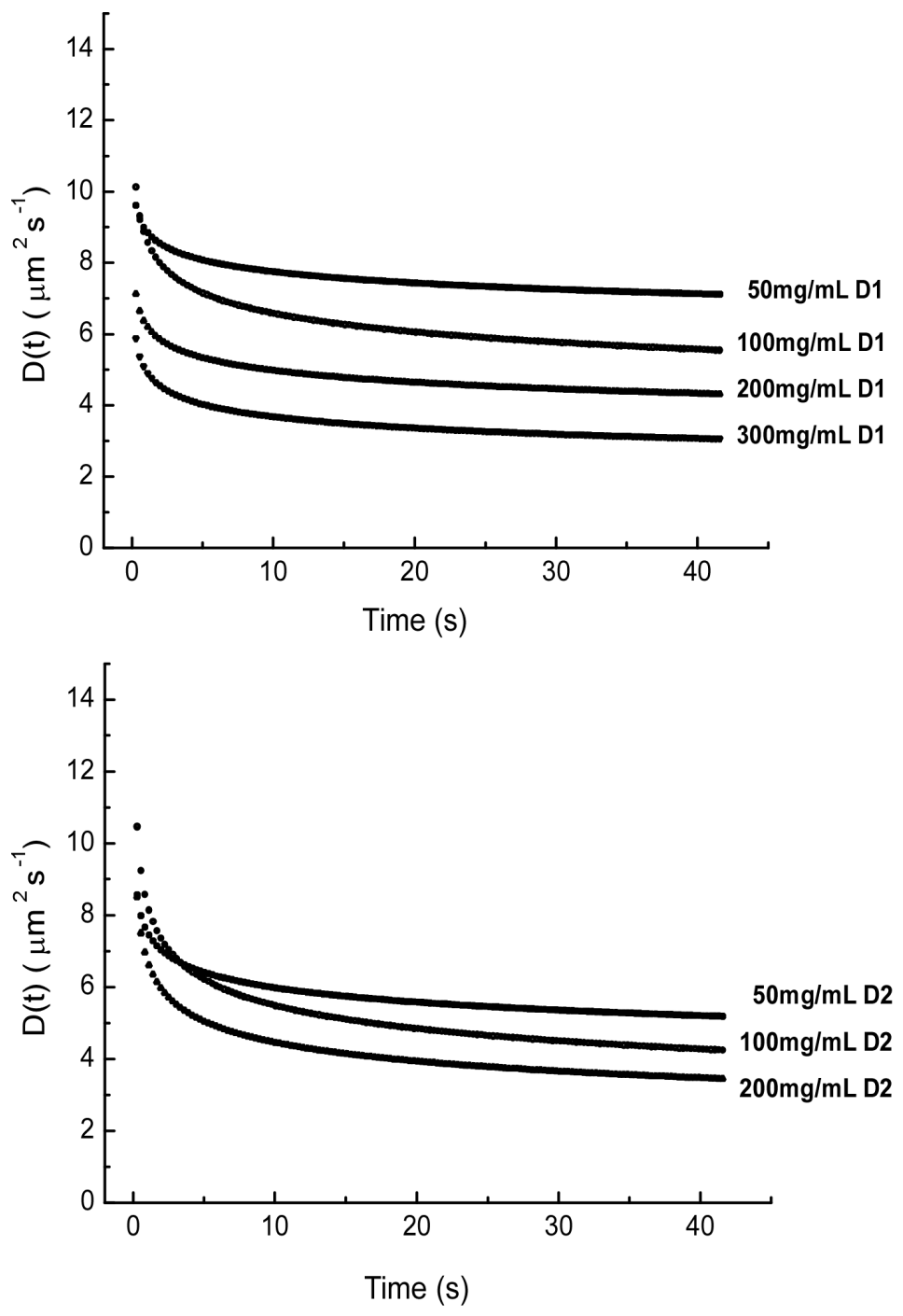